\title{
CHEMICAL ABUNDANCES AND MODELS OF WR STARS AND BLUE SUPERGIANTS
}

\author{
André Maeder \\ Geneva Observatory \\ CH-1290 Sauverny, Switzerland
}

\begin{abstract}
Firstly, a brief account is given on the recent progresses in model assumptions, which influence the evolution of surface abundances in massive stars. A concise overview of this chemical evolution is presented.

We discuss in detail the surface chemistry of WN and WC stars, in particular, the effects of the initial metallicity $\mathbf{Z}$ and mass loss rates on the resulting abundances. The abundances in WN stars are not very sensitive to model assumptions and are mainly a test on nuclear cross sections. Conversely, the chemical abundances in WC stars are very much model dependent. Major differences in the composition of WC stars (and subtype distributions) are expected according to their initial metallicity. The various models proposed to explain the N/C enrichments in blue supergiants and the properties of the progenitor of SN 1987A are reviewed.
\end{abstract}

\section{INTRODUCTION}

In astrophysical modelisations a continuous updating of model ingredients is necessary, otherwise the models soon become obsolete, (which they will do in any case). A critical comparison (cf. Maeder, 1990a) of several recent set models of low and intermediate mass stars, currently used for comparison with cluster sequences, has shown that many sets are still resting on some ancient physical data. This inspires us the reflection that, while a good observation may live forever, the lifetimes of astrophysical models are generally much shorter, since new and hopefully better data are expected soon. However, it is to be hoped that around the time they are made the new models will help to make a step forward in the understanding of stellar physics: by the discrepancies with Nature they will be able to show, the models will lead to further progresses in our knowledge and thus contribute themselves to their own obsolescence.

Among the various physical ingredients, the nuclear reaction rates, the treatment of convection, the mass loss rates, the uncertain mixing and diffusion processes may all influence the surface abundances. Thus, the spectroscopic abundance determinations may in turn place severe constraints on these processes which greatly intervene in the evolution of massive stars. This has a large astrophysical impact, since our understanding of massive star evolution is of key importance for the study of the distributions of massive stars in galaxies, for the proper identification of the different kinds of supernova progenitors and for the calculation of nucleosynthetic yields. 


\section{ON THE MAIN ASSUMPTIONS INFLUENCING CHEMICAL ABUNDANCES DURING MASSIVE STAR EVOLUTION.}

Let us briefly describe some recent and necessary improvements which greatly influence both, the chemical abundances and the course of evolution for massive stars. Firstly, the initial chemical abundances have to be the appropriate ones (cf. Grevesse, this meeting). For non-solar metallicities $\mathrm{Z}$, non-solar ratios of $\mathrm{O} / \mathrm{Fe}, \alpha$ nuclei/Fe, $\mathrm{Na} / \mathrm{Fe}, \mathrm{Al} / \mathrm{Fe}$ etc.. have to be accounted for (cf. Lambert, 1987; Wheeler et al., 1989). The initial helium abundances in the models and opacity tables can be defined according to a relation $Y(Z)=$ $0.24+\left(\frac{\Delta Y}{\Delta Z}\right) \mathrm{Z}$ where the value 0.24 corresponds to the current value of cosmological helium (cf. Audouze, 1986). The observed relative enrichment $\Delta Y / \Delta Z$ of helium to metals, ranges from about 1 to 5.7 (cf. Pagel et al., 1986). For that ratio we may take a value of 2, which also has the advantage to give a value of $Y=0.28$ for $\mathrm{Z}=0.02$, as required by solar abundances.

Of course, the opacity tables must correspond exactly to these various initial abundances. Many groups achieve that by using the Los Alamos Opacity Programme (cf. Huebner et al., 1977), for computing their own appropriate tables. For this purpose, new and complete tables have been computed at the Geneva Observatory for $Z=0.002,0.005$, 0.020 and 0.040 .

Controversies about the cross-sections of the reaction $\left.{ }^{12} \mathrm{C}(\alpha, \gamma)\right)^{16} \mathrm{O}$ have existed for several decades; let us examine the situation over recent years. New experimental determinations by Kettner et al. (1982) led to an enhancement of the reaction rate by a factor 3 to 5 with respect to the older classical value (cf. Fowler et al., 1975). New experiments by Redder et al. (1987) confirmed a cross section higher by a factor 3 to 4 for ${ }^{12} \mathrm{C}(\alpha, \gamma)^{16} \mathrm{O}$ with respect to the 1975 value. The new analysis by Plaga et al. (1987) also supported this result. On the contrary, the last compilation of data by Caughlan and Fowler (1988) again gives rates very close to the low 1975 value. However, new experimental measurements by Kremer et al. (1988) essentially confirm the high cross section by Kettner et al. (1982). The comparison of the abundance observations of SN 1987A with computations of explosive nucleosynthesis shows (cf. Thielemann et al., 1990) that the old and recent values of the cross section of ${ }^{12} \mathrm{C}(\alpha, \gamma){ }^{16} \mathrm{O}$ by the Fowler's group are clearly too low. Thus, it appears justified to keep the lower bound of the enhancement factor found by Kettner et al. (1982), Redder et al. (1987), Plaga et al. (1987) and Kremer et al. (1988). For the rate of ${ }^{14} \mathrm{~N}(\rho, \gamma)^{15} \mathrm{O}$, the slowest reaction in the CNO cycles, it is of importance to take into account the redution by a factor 1.75 according to Schröder et al. (1986). These two reactions significantly change the lifetimes and overall evolutionary properties. For other more specific reactions, the reader may refer to Arnould (this volume).

A set of detailed comparisons, between theoretical isochrones and cluster sequences in the colour-magnitude diagram of clusters and associations, has been performed recently (cf. Meynet et al. 1990; Maeder, 1990a). They confirm remarkably well the preference to be given to models with core overshooting. The distance of overshooting, i.e. the extension of full mixing beyond the formal core, appears to be about $0.25 \mathrm{Hp}$. However, it must be emphasized that the exact mixing mechanism, by which the cores are extended, is still unknown. Speaking in terms of overshooting may just be a rough simplification for much more sophisticated mixing processes. Overshooting clearly favours an earlier appearance of elements processed by CNO cycles. However, the nature of the exact mixing process may also influence the change of surface abundances (cf. also Lyubimkov, this volume). 
Mass loss by stellar winds is of paramount importance for the evolution of surface abundances in massive stars. There are presently no satisfactory stellar wind models valid for stars beyond the main sequence and thus observed mass loss rates $\dot{M}$ are generally used throughout the HR diagram. Recent data analyzes by de Jager et al. (1988) offer the most complete description of the $\dot{M}$-rates. The available data on mass loss rates apply to stars in the solar neighbourhood with $\mathrm{Z}=\mathbf{0 . 0 2}$. What is the situation for other metallicities ? The models by Brunish and Truran (1982a, b) had effectively larger mass loss rates at low metallicities and such a behaviour is rather doubtful. For non-solar metallicities, a possibility is to take the data by de Jager et al. (1988), scaled according to a relation $\left(\dot{\mathrm{M}}_{z} / \dot{\mathrm{M}}_{z_{0}}\right)$ $=\left(\mathrm{Z} / \mathrm{Z}_{0}\right)^{\xi}$. The published stellar wind models of O-stars by Kudritzki et al. (1987) give a value $\xi \cong 0.5$. For supergiants and especially red supergiants there is unfortunately no reliable information (and probably not before long) on the way the $\dot{M}$-rates may change with $\mathrm{Z}$. Models with $\xi=0.25$ and 1.00 have been performed and the comparisons with star number ratios in the LMC and SMC do also favour the choice of $\xi=0.5$ (cf. Maeder, $1990 \mathrm{c}$ ). It is to be hoped that such comparisons may provide tight constraints on the $\xi$ value.

The mass loss rates of WR stars is another story. Up to now, the models generally used the most straightforward procedure, that is to take the average observed mass loss rates of the WR stars especially more than these rates do not exhibit any clear dependence with the WR subtypes (cf. Conti, 1988). However, as shown by Schmutz et al. (1989), this leads to the troublesome result that the predicted WR luminosities are much higher than the observed values. Since there is a mass-luminosity relation for WR models (cf. Maeder, 1983; Langer, 1989a), this implies that the actual masses of most of the Wolf-Rayet stars are much smaller than predicted. This, in turn, indicates that the mass loss rates should be larger at least in some parts of the WR stages. In particular, there are several indications in favour of $\dot{M}$-rates for WNE and WC stars depending on the actual masses of WR stars (cf. Abbott et al., 1986; Langer, 1989b). The following relationship was established by Langer (1989b)

$$
\dot{M}_{W R}=(0.60-1.00) \cdot 10^{-7}\left(\frac{M_{W R}}{M_{\odot}}\right)^{2.5}
$$

where the first numerical coefficient applies to WNE and the second one to WC stars. St. Louis et al. (1988) also find a similar relation having a lower power (i.e. an exponant $\sim 1.3$ ) for the $\dot{M}$ vs. $M$ relation for WR stars in binaries. The exponant 2.5 is also well supported by the analysis of Smith and Maeder (1989), when the WNL stars are removed (cf. Langer, 1989b). The study of wind properties in He-stars by Bandiera and Turolla (1990) shows a relation of the form $\dot{M}$ vs. $M^{2.3}$. For late WN stars (WNL), there is at the moment no evidence of a similar relation and thus the average $\dot{\mathrm{M}}$-rate of $4.10^{-5} \mathrm{M}_{\odot} y r^{-1}$ is then taken for now (cf. Conti, 1988). However, we clearly emphasize that on this point there is room for improvement in the future.

The effective temperature of Wolf-Rayet stars is also a delicate problem, since the winds may have a non-negligible optical thickness. One may adopt a simple correction scheme to account for this effect (cf. de Loore et al., 1982; Langer, 1989a) in the case of electron scattering opacity. However, better procedures allowing to include the line opacities are now in development. The further major step will undoubtedly be the building of complete stellar models, with consistant winds, atmosphere and interior. The various developments, mentioned above, have been included in recent sets of models from 1.5 to $120 \mathrm{M}_{\odot}$ with 
$\mathrm{Z}=0.002,0.005,0.020$ and 0.040 (cf. Maeder, 1990b, and in prep.)

\section{EVOLUTION OF SURFACE CHEMISTRY}

The evolution of massive stars in the HR diagram, and also of surface abundances, depends very strongly on the mass loss rates, the initial mass and convection criteria. If there is a real significant scatter in the mass loss rates (say by a factor of 2 ), it may well be that for a given initial stellar mass the evolution could not be represented by a single track but rather by a family of tracks with a certain variety of $\dot{\mathrm{M}}$-rates. Presently, we are still working with average rates. This has led us to identify three main evolutionary sequences in the HR diagram (cf. Chiosi and Maeder, 1986) depending on the initial stellar masses:

\begin{tabular}{|c|c|c|}
\hline $\mathbf{M}$ & $\geq$ & $\begin{array}{ll}\mathrm{M}_{1} & \text { always blue } \\
\text { O-star - Of - BSG - LBV - WR - SN (Type Ib) }\end{array}$ \\
\hline $\mathrm{M}_{1}$ & $>$ & $\begin{array}{l}M \geq M_{2} \text { blue - red - blue } \\
\text { O-star - BSG - RSG - }(\mathrm{OH} / \mathrm{IR})-\mathrm{WR}-\mathrm{SN}(\mathrm{Type} \mathrm{Ib})\end{array}$ \\
\hline $\mathbf{M}$ & $\geq$ & $\begin{array}{ll}\mathrm{M}_{2} & \text { blue -red - blue -red } \\
\text { O-star - } & \text { RSG - YSG - BSG - RSG - SN (Type II) } \\
& \text { (Cepheid loop) }\end{array}$ \\
\hline
\end{tabular}

In addition different scenarios have been suggested in connexion with SN 1987A (cf. Langer et al. (1989).

$$
\text { O-star - RSG - YSG - BSG - RSG - BSG - SN (1987A) }
$$

In the Milky Way, the initial mass $M_{1}$ seems to be around $40 M_{\odot}$ and $M_{2}$ around 25 $\mathrm{M}_{\odot}$. These limiting masses and the corresponding scenarios may vary for different initial metallicities.

The evolution of surface abundances in massive stars has been studied by several authors (cf. Noels and Gabriel, 1981; Maeder, 1983, 1987, 1990b; Prantzos et al. 1986; Maeder and Meynet, 1987). Generally speaking, we may distinguish 5 stages in the evolution of surface abundances:

1. Initial cosmic abundances: $\mathrm{C} / \mathrm{N} \simeq 4, \mathrm{O} / \mathrm{N} \simeq 10$.

2. Partially, CNO processed elements, or diluted processed elements. This stage is characterized by $\mathrm{N}$-enrichment, $\mathrm{C}$ and to a smaller extent by $\mathrm{O}$-depletion, and ${ }^{13} \mathrm{C} /{ }^{12} \mathrm{C}$ enhancement. This is typical of red supergiant models.

3. CNO equilibrium, $\mathrm{H}$ present. There we have typically $\mathrm{C} / \mathrm{N} \simeq 0.02$ and $0 / \mathrm{N} \simeq 0.1$, i.e. ratios which are two orders of a magnitude below the cosmic ratios. Generally, the hydrogen contents $\mathrm{X}_{8}$ at the surface is lower than 0.40 . This composition is typical of Luminous Blue Variables and of WNL (late) stars (see below).

4. CNO equilibrium with little or no $\mathrm{H}$ left. This situation is that of WNE (early) stars.

5. Products of partial He-burning: large $\mathrm{He}, \mathrm{C}$ and $\mathrm{O}$ contents, as shown by WC and WO stars. There we may also distinguish the WCL (late) stars where the $\mathrm{C}$ and $\mathrm{O}$ contents are small, and the WCE (early) stars where the $\mathrm{C}$ and $\mathrm{O}$ contents are large (cf. Smith and Hummer, 1988, see below). In WO stars, the abundance in $\mathrm{O}$ exceeds that in $\mathbf{C}$.

The above sequence is consistant with a progression in the exposition of nuclear products. It is interesting to recall that the changes in $\mathrm{C} / \mathrm{N}$ occur much faster than those in $0 / \mathrm{N}$ (cf. Figs. 1 and 2). This is due to the fact that $O N$ loops need much more time at a given 
temperature to reach equilibrium than the $\mathrm{CN}$ cycle. Of course, not all stars go through every of the 5 steps mentioned above : the smaller the initial mass, the shorter the itinerary. In the case of the WR stars, great attention must be paid to the fact that the lifetimes in the WR phases are quite variable having as well different entry and exit points according to the inital mass and metallicity $\mathrm{Z}$ (cf. Maeder, 1990c).

The evolution of the surface abundances has been computed for massive stars and various metallicities (cf. Maeder, 1990b). From such data, drawings showing the run of the various $X_{i}$ in function of the remaining mass and time can be made (cf. Figs. 3 and 4). Also, the cartography of the $\mathrm{C} / \mathrm{N}$ or $\mathrm{O} / \mathrm{N}$ ratios in the $\mathrm{HR}$ diagram can be obtained (cf. Maeder, 1987).

\section{SURFACE CHEMISTRY IN WN STAR MODELS}

The main theoretical abundance ratios in WN star models of different initial metallicities are (in mass fractions):

\begin{tabular}{|c|c|c|c|c|}
\hline & $\mathrm{Z}=0.040$ & $\mathrm{Z}=0.020$ & $\mathrm{Z}=0.005$ & $\mathrm{Z}=0.002$ \\
\hline $\mathrm{C} / \mathrm{He}$ & $(4.8-11) \mathrm{E}-4$ & $(2.6-4.4) \mathrm{E}-4$ & $(7.1-18) \mathrm{E}-5$ & $(3.4-4.1) \mathrm{E}-5$ \\
$\mathrm{~N} / \mathrm{He}$ & $(2.6-3.9) \mathrm{E}-2$ & $(1.3-1.9) \mathrm{E}-2$ & $(3.4-5.2) \mathrm{E}-3$ & $(1.4-2.0) \mathrm{E}-3$ \\
$\mathrm{C} / \mathrm{N}$ & $(1.3-3.1) \mathrm{E}-2$ & $(1.6-2.7) \mathrm{E}-2$ & $(1.8-3.5) \mathrm{E}-2$ & $(1.7-3.0) \mathrm{E}-2$ \\
$\mathrm{C} / \mathrm{O}$ & $.06-0.6$ & $0.1-1.1$ & $0.1-1.5$ & $0.4-1.8$ \\
\hline
\end{tabular}

These are the main ranges for predicted abundance ratios. The indicated ranges encompass both the WNL and WNE stages. Although there is no large difference between these two stages, the C-contents are generally about 0.2 dex larger in the WNE stage than in the WNL stage. For the $\mathrm{O}$-contents we have an opposite situation with about the same amplitude; $\mathrm{N}$ is the same in the WNL and WNE phases.

We notice of course that all these abundance ratios are highly non-solar, by at least 2 orders of a magnitude. The $\mathrm{C} / \mathrm{He}$ and $\mathrm{N} / \mathrm{He}$ ratios in WN stars have a behaviour which is essentially linear with the initial $\mathrm{Z}$ : higher initial $\mathrm{Z}$ implies higher $\mathrm{C} / \mathrm{He}$ and $\mathrm{N} / \mathrm{He}$ in the WN stages. The small deviations from a strict linearity are due to the fact that the initial abundance ratios of heavy elements have been taken non-solar; also the extension of stellar cores and the dilution factors may be slightly different for different initial $\mathrm{Z}$ and masses. As far as the $\mathrm{C} / \mathrm{N}$ and $\mathrm{C} / \mathrm{O}$ ratios are concerned, we notice that they are as expected, essentially similar in WN star models originating from different inital Z. For further abundance ratios in WN stars, the reader may refer to the detailed tables (cf. Maeder 1990b). In general, for a given initial $\mathrm{Z}$, there are very little differences between the predictions of various models for WN stars. The reason is that WN abundances are equilibrium values, which are not very much model dependent, apart from changes in the nuclear cross sections. 

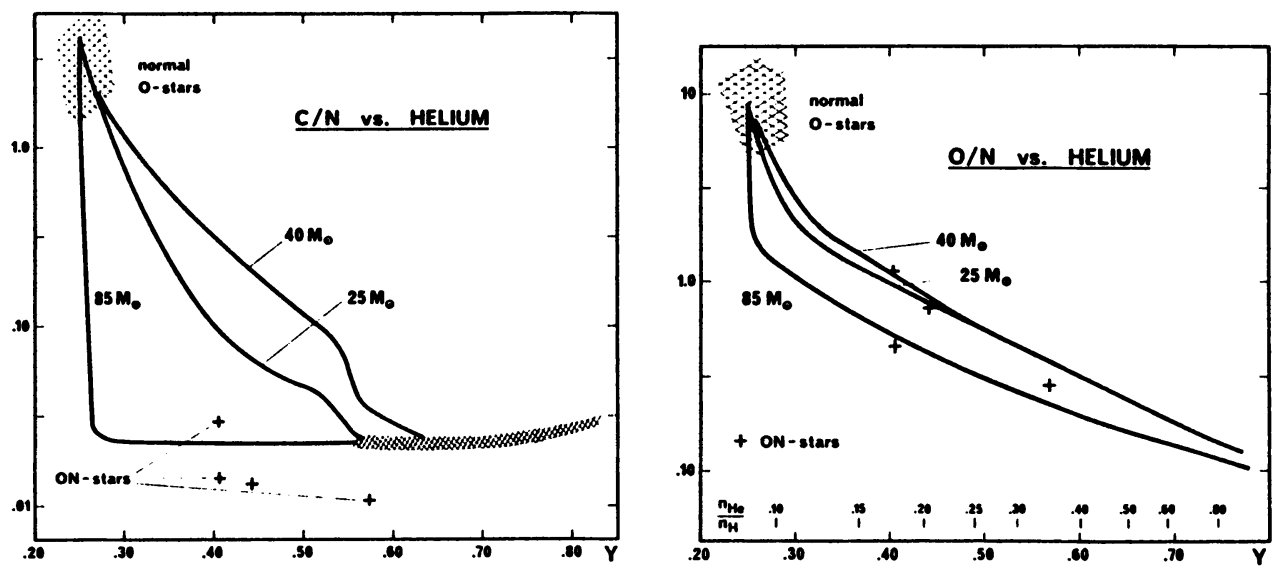

Fig. 1 and 2 : Relations between the $C / N, O / N$ surface ratios (in mass) and the surface helium content $Y$ for various initial masses $(Z=0.02)$. Crosses indicate the location of some ON stars studied by Kudritzki (1985).

For comparison with observations, it is very important to use the set of models with the appropriate inital composition. The values given by Willis (this volume) are: $\mathrm{C} / \mathrm{He}$ $=2.1 \cdot 10^{-5}-8.4 \cdot 10^{-4}, \mathrm{~N} / \mathrm{He}=3.5 \cdot 10^{-4}-1.4 \cdot 10^{-2}, \mathrm{C} / \mathrm{N}(0.6-6.0) \cdot 10^{-2}$ and $\mathrm{C} / \mathrm{O} \geq 0.02$ in mass fractions. The good agreement of these various values with the theoretical ones certainly well confirm the identification of WNL stars with bare cores in which CNO equilibrium products are visible. However, the scatter in the observations is still too large to enable us to draw further conclusions. Also, we must remember, since the abundances have their equilibrium values, that the abundances in the WN stage do not represent a very constraining test for the models. They represent, however, a very interesting test for the nuclear cross-sections and the agreement found with observations suggests that these cross-sections are correct on the whole.

\section{SURFACE CHEMISTRY IN WC STAR MODELS}

Recent evolutionary models, including the mass loss rate dependence on metallicity and also the $\dot{M}$ vs. $M$ relation in WNE and WC stars, predict the following abundance ratios in WC stars:

\begin{tabular}{|c|c|c|c|c|}
\hline mass ratio & $\mathrm{Z}=0.040$ & $\mathrm{Z}=0.020$ & $\mathrm{Z}=0.005$ & $\mathrm{Z}=0.002$ \\
\hline $\mathrm{C} / \mathrm{He}$ & $.03-2.5$ & $0.03-2.5$ & $0.04-3.4$ & $0.6-45$ \\
$\mathrm{O} / \mathrm{C}$ & $0.1-1.5$ & $0.12-2.3$ & $1.3-7.2$ & $3-90$ \\
\hline
\end{tabular}


We notice many interesting points in this table.

1) At a given initial $Z$, there is a very large range, i.e. by 2 orders of a magnitude, in the predicted $\mathrm{C} / \mathrm{He}$ ratios. The reason is that in $\mathrm{WC}$ stars we see products of partial He-burning. At the beginning of the WC phase, we see products in an early stage of nuclear processing, henceforth the small $\mathrm{C} / \mathrm{He}$ ratios. At the end of the WC phase, products in an advanced stage of $\mathrm{He}$-burning are visible and thus the $\mathrm{C} / \mathrm{He}$ ratios are large.

2) For the same reasons, the range of the predicted $\mathrm{O} / \mathrm{C}$ ratios for $\mathrm{WC}$ stars is also wide.

3) For lower initial $\mathrm{Z}$ values, we notice that $\mathrm{WC}$ stars have high ranges in the $\mathrm{C} / \mathrm{He}$ and $\mathrm{O} / \mathrm{C}$ ratios. This trend is also beautifully illustrated by the comparison of WC abundances in Fig. 3 and 4. The reasons for the much larger $\mathrm{C} / \mathrm{He}$ and $\mathrm{O} / \mathrm{C}$, is that for low $\mathrm{Z}$ models the products of He-burning appear (if they do it !) only at a very advanced stage of nuclear processing due to the much smaller mass loss rates in previous evolutionary phases. This is a key effect which determines the main trend for the distribution of WC stars in galaxies of different $\mathrm{Z}$. Another related consequence is, that the relative frequency of WC stars is much inferior at lower $\mathrm{Z}$. Detailed number statistics have been established (cf. Maeder, 1990c).

Contrarily to the case of WN stars, the chemistry of WC star models is extremely sensitive to model assumptions. Changes in the mass loss rates make important differences. Also, mixing processes and overshooting produce differences in $\mathrm{C} / \mathrm{He}$ and $\mathrm{O} / \mathrm{C}$ ratio in WC stars as shown by the comparison of the models by Maeder and Meynet (1987) and by Prantzos et al. (1986). Models with large overshoot produce lower $\mathrm{C} / \mathrm{He}$ and $\mathrm{O} / \mathrm{C}$ ratios at the beginning of the WC phase, since large cores favour an early appearance of the products of nuclear burning.

Let us compare the model results with observations. The values found for the $\mathrm{C} / \mathrm{He}$ ratios are $0.3-0.6$ (Nugis, 1982), $0.02-0.1$ (Smith and Willis, 1982), $0.1-3$ (Smith and Hummer, 1988), 0.4 - 2.5 (Torres, 1988) and Hillier (1989) finds a value of 1.5 for a WC5 star. Willis (this conference) gives $\mathrm{C} / \mathrm{He}=0.12-2.0, \mathrm{O} / \mathrm{C} \leq 0.3$ (in mass fractions). We notice a good agreement with the model predictions. In the future, if a large number of observed values are available, it may be useful to compare also the theoretical and observed abundance distributions. Presently, we may conclude that the above agreement confirm the proper identification of WC stars as bare cores in which the products of He-burning are visible at different stages of nuclear processing.

Smith and Hummer (1988) have determined the $\mathrm{C}^{+4} / \mathrm{He}^{++}$ratio in WC stars and have shown that it varies with the WC subtypes. The ionisation potential of the two ions are rather similar, thus they should dominate over the same volume. Moreover, in the hydrogenic approximation $\mathrm{C}^{+4} / \mathrm{He}^{++}$and $\mathrm{O}^{+4} / \mathrm{He}^{++}$cannot be separated and thus the observed value of $\mathrm{C}^{+4} / \mathrm{He}^{++}$constitutes a direct estimate of the $(\mathrm{C}+\mathrm{O}) / \mathrm{He}$ ratio. The following calibration is derived (cf. Smith and Hummer, 1988; Smith and Maeder, 1990):

$\begin{array}{ll}\text { WC 9: } & (\mathrm{C}+\mathrm{O}) / \mathrm{He}=0.03 \text { to } 0.06 \text { (in numbers) } \\ \text { WC 8: } & (\mathrm{C}+\mathrm{O}) / \mathrm{He}=\sim 0.1 \\ \text { WC 7: } & (\mathrm{C}+\mathrm{O}) / \mathrm{He}=\sim 0.2 \\ \text { WC 6: } & (\mathrm{C}+\mathrm{O}) / \mathrm{He}=\sim 0.3 \\ \text { WC 5: } & (\mathrm{C}+\mathrm{O}) / \mathrm{He}=\sim 0.55 \\ \text { WC 4: } & (\mathrm{C}+\mathrm{O}) / \mathrm{He}=0.7 \text { to } 1.0 \\ \text { WO : } & (\mathrm{C}+\mathrm{O}) / \mathrm{He} \quad \text { greater than } 1.0\end{array}$




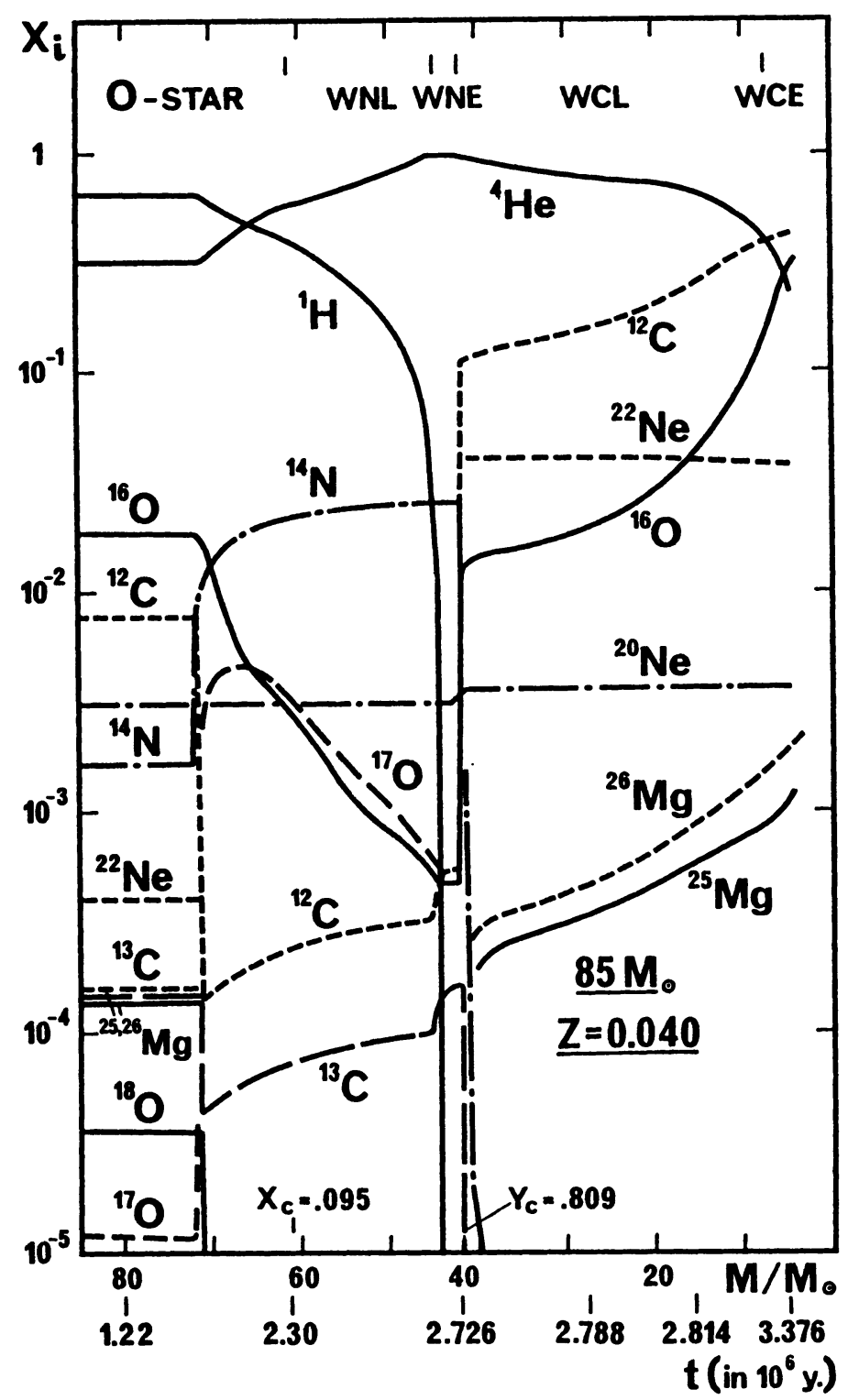

Fig. 3 : Changes of surface abundances (in mass fraction) in terms of the remaining mass for the model with an initial mass of $85 M_{\odot}$ and $Z=0.040$. The ages are also given on the lower axis. The corresponding evolutionary status is indicated at the top. 


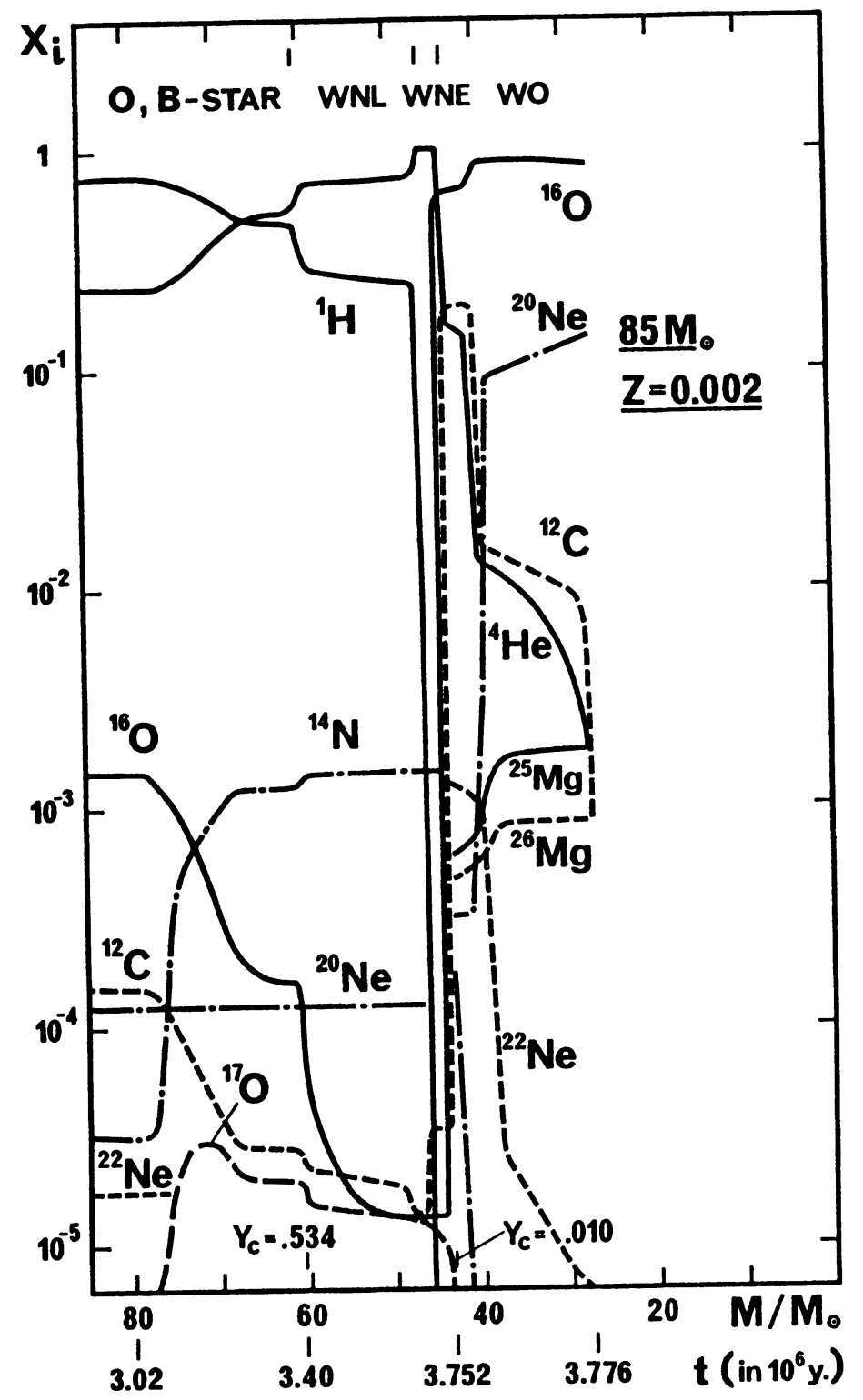

Fig. 4 : Same as Fig. 3 for the model with an initial mass of $85 M_{\odot}$ and $Z=0.002$. 
Since, as seen above, low $\mathrm{C} / \mathrm{He}$ and $\mathrm{O} / \mathrm{C}$ ratios occur preferably in high $\mathrm{Z}$ regions and conversely, this means that late WC stars should be relatively more frequent in low $\mathrm{Z}$ regions. This number statistic has been studied by Maeder (1990 c).

The case of neon is delicate and interesting. The models (cf. Figs. 3 and 4 ) show that at high $\mathrm{Z}$, the (usually) main isotope ${ }^{20} \mathrm{Ne}$ has an abundance that is not very different from the cosmic value since the nuclear processing in the chain $\alpha(2 \alpha, \gamma)^{12} \mathrm{C}(\alpha, \gamma)^{16} 0$ $(\alpha, \gamma)^{20} \mathrm{Ne}$ is not very much advanced. Conversely, the isotope ${ }^{22} \mathrm{Ne}$, which is related to CNO elements, is very abundant (a factor of $10^{2}$ with respect to the cosmic value). This situation, at low $\mathrm{Z}$, is quite different. The He-processing is so much advanced when WC stars are formed, that the ${ }^{20} \mathrm{Ne}$-content may reach $\mathrm{X}\left({ }^{20} \mathrm{Ne}\right) \simeq 0.1$, a value three orders of magnitude greater than the local cosmic value! Simultaneously, the isotope ${ }^{22} \mathrm{Ne}$ is destroyed by the reactions ${ }^{22} \mathrm{Ne}(\alpha, \gamma){ }^{26} \mathrm{Mg}$, which also produce s-elements.

The models predict $\mathrm{Ne} / \mathrm{He}$ ratio greater than 0.03 (in mass fraction, cf. Figs. 3 and 4). However, the infrared observations by Barlow et al. (1988), for the WC8 star $\gamma$ Vel give $\mathrm{Ne} / \mathrm{He}=0.005$ (in mass fraction), i.e. at least 6 times less than predicted, (however, this is twice the solar ratio). Thus, the question is: in which form are the CNO ashes ? This must be either in the form of ${ }^{14} \mathrm{~N},{ }^{18} \mathrm{O},{ }^{22} \mathrm{Ne}$ or ${ }^{25,26} \mathrm{Mg}$, in view of the existing reaction chains. There is neither evidence of $\mathrm{N}$ in WC stars (see Willis, this volume) nor of conspicuous $\mathrm{Mg}$ lines while $\mathrm{Ne}$ is missing. Thus, we make the suggestion that the CNO ashes could be in some WC stars (mainly WCL) hidden in the form of ${ }^{18} \mathrm{O}$, which would of course be undistinguishable of the main bulk of ${ }^{16} \mathrm{O}$. This would imply that some nuclear cross sections have to undergo significant changes, a fact which is quite possible according to Arnould (this volume).

Let us also mention that the study of the number ratios of WNL, WNE, WCL and WCE stars, in regions of different metallicities, offer a very important test for the models (cf. Maeder, 1990c). The lifetimes in the various WR phases have been tabulated and may be used to calculate, for given initial mass functions (IMF) and star formation rates (SFR) the theoretically expected numbers of the various types of WR stars. The theoretical numbers obtained with a standard IMF and a constant SFR compare quite well with the observed number ratios at various galactocentric distances in the Milky Way and in galaxies of different $Z$ in the Local Group (cf. Smith, 1988) namely M 31, M 33, LMC, SMC, NGC 6822 and IC 1613. Great care must be given that the numbers obtained do not necessarily apply for a single HII region, where aging effects are likely to intervene.

\section{BLUE SUPERGIANTS: QUESTIONS AND MODELS RAISED BY CNO OBSERVATIONS AND SN 1987A.}

Let us start by mentionning a few observational facts. Among blue supergiants, there are evidences of normal (or solar) abundances as well as evidences of partial CNO processing. Dufton and Lennon (1989) have shown that two blue supergiants with the same gravity and $\mathrm{T}_{\text {eff }}$ (around type B 1.5 Ia) show different compositions, one being solar, the other one exhibiting an He excess and an increase in $\mathrm{N}$ by a factor of 6 . Walborn $(1976,1988)$ has forwarded the hypothesis that ordinary OB supergiants are enriched in CNO processed elements, while the so called $O B C$ stars just have the cosmic abundances. Observations in support of this claim were given. The same kind of finding is presented in the result published by Kudritzki $(1987,1990)$ for B-supergiants in the LMC, some ones showing an 
excess of nitrogen. Further evidences for LMC and SMC supergiants were also provided by Reitermann et al. (1990).

The thunder in the sky of blue supergiant models was of course SN 1987A and the evidence that its blue progenitor had a $\mathrm{N} / \mathrm{C}$ ratio of about 8.8, i.e. a factor of 30 more than the solar ratio (cf. Fransson et al., 1988). After SN 1987A another explosion, i.e. in the number of proposed models, occured with the aim of explaining these properties. Let us shortly mention some of these models with their respective successes and failures.

- 1. Models with low $\mathrm{Z}$, without mass loss: (cf. Hillebrandt et al. 1987) finish their life in the blue, however they produce no red supergiants and show no N/C enhancements, in contradiction with observations.

- 2. Models with low $\mathrm{Z}$, having mass loss: may end their life in the blue as a result of mass loss (cf. Wood and Faulkner, 1987; Maeder, 1987b) only if the envelope mass is smaller than $1 \mathrm{M}_{\odot}$, which is not supported by the interpretation of the light curve (Shigeyama et al. 1988; Woosley, 1988).

- 3. Models with the Ledoux criterion for convection have been proposed by Woosley (1988) to account for the blue progenitor, (cf. also Weiss, 1989). However, when a moderate mass loss is taken into account, the final blue location occurs too early, i.e. during central He-burning, which is not in agreement with the suggestion that the precursor of SN 1987A was a red supergiant a few thousand years before the explosion.

- 4. Models with semiconvetion, being intermediate between the Ledoux and Schwarzschild criterion, have been proposed by Langer et al. (1989) to account for the blue SN progenitor. For some fine tuning of the convection parameter, this treatment well produces the blue progenitor. However, it does not simultaneously account for the observed $\mathrm{He}$ and N/C enrichment in SN 1987A. An additional mixing or diffusion process has then to be called for to explain these surface enrichements. Langer et al. (1989) are using there rotational diffusion, as proposed by Maeder (1987c) to explain some of the $\mathrm{ON}$ blue stragglers in young associations. If this applies also to SN 1987A, this means that two fine tunings are required to explain simultaneously the blue location and the $\mathrm{N} / \mathrm{C}$ enrichment. This is not impossible, but this also means that the generality of that solution can be questioned.

- 5. Models with late mixing: the possibility of mixing of the external layers of a red supergiant at the end of the He-burning phase has been envisaged by Saio et al. (1988). Indeed, our models also confirm that at this stage, the external and intermediate convective zones become extremely close to each other, so that any tiny convective extension is likely to bring the 2 zones into contact. The resulting mixing produces simultaneously the He- and N/C enrichements as well as the blue location of the SN progenitor and this makes the suggestion by Saio et al. (1988) an attractive one.

In conclusion of this discussion, we see that presenlty there is not one unique interpretation. possible. My personal view is, that the sensitivity of the supergiant phases to opacity and uncertain mixing processes are so important that until these effects are not better clarified, for example by more studies on the abundances, we may not be able to get the right interpretation for the blue location and composition of the Supernova progenitor.

Let us finally note that the properties of the WR stars are much less sensitive to these uncertainties in convection treatments and mixing processes, since at the WR stage, most of the concerned layers have already been taken away by mass loss. 


\section{REFERENCES}

Abbott D., Bieging J.H., Churchwell E., Torres A.V.: 1986, Astrophys. J. 303, 239

Audouze J.: 1986, in "Nucleosynthesis and Chemical Evolution", 16th Saas-Fee course, Ed. Geneva Observatory, p. 431

Bandiera R., Turolla R.: 1990, Astron. Astrophys. 231, 85

Barlow M.J., Roche P.F., Aitken D.K.: 1988, M.N.R.A.S. 232, 821

Brunish W.M., Truran J.W.: 1982a, Astrophys. J. 256, 247

Brunish W.M., Truran J.W.: 1982b, Astrophys. J. Suppl. Ser. 49, 447

Caughlan G.R., Fowler W.A.: 1988, At. Nucl. Data Tables 40, 283

Chiosi C., Maeder A.: 1986, Ann. Rev. Astron. Astrophys. 24, 329

Conti P.S.: 1988, in "O stars and Wolf Rayet stars", NASA SP-497.Eds. P.S. Conti and A. B. Underhill, p.81

Dufton P.L., Lennon D.J.: 1989, Astron. Astrophys. 211, 397

Fransson C., Cassatella A., Gilmozzi R., Kirshner R., Panagia N., Sonneborn, G., Wamsteker W.: 1988, Astrophys. J. 336, 429

Fowler W.A., Caughlan G.R., Zimmermann B.A.: 1975, Ann. Rev. Astron. Astrophys. 13,69

Hillebrandt W., Höflich P., Truran J.W., Weiss A.: 1987, Nature 327, 597

Hillier D.J.: 1989, Astrophys. J. 347, 392

Huebner W.F., Merts, A. L., Magee, W. H.jr. Argo, M. F.: 1977, Los Alamos Sci. Lab. Report LA-6760 M UC-346, Astrophysical Opacity Library

de Jager C, Nieuwenhuijzen H., van der Hucht K.A.: 1988, Astron. Astrophys Suppl. Ser. 72, 259

Kettner K.U., Becker H.W., Buchmann L., Görres J., Kräwinkel H., Rolfs C., Schmalbrock P., Trautvetter H.P., Vlieks A.: 1982, Z. Phys. A 308, 73

Kremer R.M., Barnes C.A., Chang K.H., Evans H.C., Filipoone B. W., Hahn K. H., Mitchell L. W.: 1988, Phys. Rev. Lett. 60, 1475

Kudritzki R.P.: 1985, in "Production and distribution of CNO elements", ESO Workshop, Eds. I. J. Danziger et al., p. 277

Kudritzki R.P.: 1990, in "Massive Stars in Starburst", STScI Workshop, Ed. C. Leitherer in press

Kudritzki R.P., Groth H.G., Butler K., Husfeld D., Becker S., Ebner F., Fitzpatrick E.: 1987 , in "SN 1987A", ESO Workshop n ${ }^{\circ} 26$, Ed. I.J. Danziger, p. 39

Kudritzki R.P., Pauldrach A., Puls J.: 1987, Astron. Astrophys. 173, 293

Lambert D.: 1987, J. Astrophys. Astron. 8, 103

Langer N.: 1989a, Astron. Astrophys. 210, 93

Langer N.: 1989b, Astron. Astrophys. 220, 135

Langer N., El Eid M. F., Baraffe I.: 1989, Astron. Astrophys. 224, L17

de Loore C., Hellings P., Lamers H.J.G.L.M.: 1982, in "Wolf-Rayet Stars: observation, physics, evolution", IAU Symp. 99, Eds. C. de Loore and A.J. Willlis (Reidel Publ.) p. 53

Maeder A.: 1983, Astron. Astrophys. 120, 113

Maeder A.: 1987, Astron. Astrophys. 173, 247

Maeder A.: 1987b, in "SN 1987A", ESO Workshop, Ed. I.J. Danziger, p. 251

Maeder A.: 1987c, Astron. Astrophys. 178, 159

Maeder A.: 1990a, in "Astrophysical ages and dating methods" Ed. E. Vangioni-Flam et al. (Editions Frontières), p. 71

Maeder A.: 1990b, Astron. Astrophys. Suppl. Ser. 84, 139

Maeder, A.: 1990 c, Astron. Astrophys., in press

Maeder, A., Meynet G.: 1987, Astron. Astrophys. 182, 243

Meynet, G., Mermilliod J. C., Maeder A.: 1990, in "Astrophysical ages and dating methods" Ed. E. Vangioni-Flam et al. (Editions Frontières), p. 91

Noels A., Gabriel M.: 1981, Astron. Astrophys. 101, 215 
Nugis T.: 1982, in "WR Stars: observation, physics, evolution" IAU Symp. 99, Eds. C. de Loore and A.J. Willis (Reidel Publ.) pp. 127, 131

Pagel B.E.J., Terlevich R., Melnick J.: 1986, PASP 98, 1005

Plaga R., Becker, H.W., Redder A., Rolfs C., Trautvetter H.P.: 1987, Nuclear Physics A465, 291

Prantzos, N., Doom, C., Arnould, M., de Loore, C.: 1986, Astrophys. J. 304, 695

Redder A., Becker H.W., Rolfs C., Trautvetter H.P.: 1987, Nuclear Phys. A462, 385

Reitermann A., Baschek B., Stahl O., Wolf B.: 1990, Astron. Astrophys. 234, 109

Saio H., Nomoto K., Kato M.: 1988, Nature 334, 508

Schmutz W., Hamann W.-R., Wessolowski K.: 1989, Astron. Astrophys. 210, 236

Schröder U., Becker H.W., Görres J., Rolfs C., Trautvetter, H. P., Azuma, R. E., King, J.: 1986, Proceedings of the NATO Adv. Research Workshop, (5th Moriond Astrophys. Meeting) on "Nucleosynthesis and its implications on nuclear and particle physcis" Ed. Reidel, Dordrecht p. 431

Shigeyama T., Nomoto K., Hashimoto M.: 1988, Astron. Astrophys. 196, 141

Smith L. F.: 1988, Astrophys. J. 327, 128

Smith L. F., Hummer D.G. : 1988, M.N.R.A.S. 230, 511

Smith L. F., Maeder A.: 1989, Astron. Astrophys. 211, 71

Smith L. F., Maeder A.: 1990, Astron. Astrophys., in press

Smith L. F., Willis A.J.: 1982, Monthly Notices Roy. Astron. Soc. 201, 451

St. Louis N., Moffat A.F.J., Drissen L., Bastien P., Robert C.: 1988, Astrophys. J. 330, 286

Thielemann F.K., Hashimoto M., Nomoto K.: 1990, Astrophys. J., 349, 222

Torres A.V.: 1988, Astrophys. J. 325, 759

Walborn N.: 1976, Astrophys. J. 205, 419

Walborn N.: 1988, in "Atmospheric Diagnostics of Stellar Evolution", IAU Coll. 108, Ed. K. Nomoto (Springer Verlag), p. 70

Weiss A.: 1989, Astrophys. J. 339, 365

Wheeler J.C., Sneden C., Truran J.W.: 1989, Ann. Rev. Astron. Astrophys. 27, 279

Wood P.R., Faulkner D.J.: 1987, Proc. Astron. Soc. Austr. 7, 75

Woosley S.E.: 1988, Astrophys. J. 330, 218 